\title{
Crystal Splitting in the Growth of $\mathrm{Bi}_{2} \mathrm{~S}_{3}$ \\ Materials Sciences Division, Lawrence Berkeley National Laboraotry, and Department of Chemistry, University of California, Berkeley, CA 94720 \\ Jing Tang and A. Paul Alivisatos*
}

\begin{abstract}
Novel $\mathrm{Bi}_{2} \mathrm{~S}_{3}$ nanostructures with a sheaf-like morphology are obtained via reaction of bismuth acetate-oleic acid complex with elemental sulfur in 1-octadecence. We propose these structures form by the splitting crystal growth mechanism, which is known to account for the morphology some mineral crystals assume in nature. By controlling the synthetic parameters, different forms of splitting, analogous to observed in minerals, are obtained in our case of $\mathrm{Bi}_{2} \mathrm{~S}_{3}$. These new and complex $\mathrm{Bi}_{2} \mathrm{~S}_{3}$ nanostructures are characterized by TEM, SEM, XRD and ED.
\end{abstract}

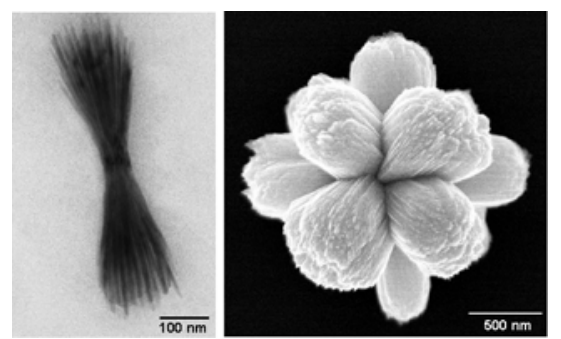

Table of Content Figure

* To whom correspondence should be addressed. Email address: alivis@berkeley.edu. 
Synthesis of nanoscaled inorganic crystals with controlled size, shape and hierarchy has attracted intensive research interest as they are potential building blocks for advanced materials and devices with designed functions in areas as diverse as electronics, optics, catalysis and ceramics [1]. With deeper understanding of crystal growth kinetics, nanocrystals with complex forms evolving from tetrapods [2], inorganic dendrimers of precisely controlled generations [3], to hyperbranched nanostructures [4] have been successfully created by the colloidal solution method. Meanwhile, in a strongly related field, bio-inspired morphosynthesis using designed artificial organic templates offers another approach in generating inorganic crystals with unusual structure specialty and complexity [5]. For example, in the case of $\mathrm{BaSO}_{4}$, a variety of morphologies including cones, multiple-cones [6], peanuts and flowers with a crystallographically forbidden ten-fold symmetry [7] as well as complex bundles and superstructures [8] have been obtained by utilizing various double hydrophilic block copolymers as crystal modifiers.

Of particular interest here is the range of mechanisms by which it is possible to make branched nanocrystals. Polytpism between hexagonal and cubic structures was exploited to create branch points in semiconducting nanocrystals [2-3]. Changes in relative growth rates of two facets are responsible for branching in those nanocrystals. Natural systems show branching by a mechanism of 'crystal splitting', and the goal of this work is to demonstrate this mechanism can be used to prepare artificial branched nanocrystals.

$\mathrm{Bi}_{2} \mathrm{~S}_{3}$ belongs to the family of main group metal chalcogenides $\mathrm{A}_{2}{ }^{\mathrm{V}} \mathrm{B}_{3}{ }^{\mathrm{VI}}$ ( $\mathrm{A}=\mathrm{As}$, Sb and $\mathrm{Bi}$, $\mathrm{B}=\mathrm{S}$, Se and Te), an important class of semiconductors with numerous applications including photovoltaics [9] and thermoelectrics [10]. Recently $\mathrm{Bi}_{2} \mathrm{~S}_{3}$ nanoparticles have found new applications as imaging agents in x-ray computed tomography [11]. Simple one-dimensional $\mathrm{Bi}_{2} \mathrm{~S}_{3}$ nanostructures such as nanorods, nanowires and nanoribbons have been made by colloidal solution methods [12], solventless arrested precipitation [13], and solvothermal or hydrothermal decomposition [14]. More complex forms of $\mathrm{Bi}_{2} \mathrm{~S}_{3}$ such as nanoflowers [15-16] and snowflakes 
[17] have been made by employing the soft template effect of an ionic liquid solution [15], polymers [16] or biomolecules [17], respectively.

In this paper, we used a simple colloidal solution method and synthesized novel $\mathrm{Bi}_{2} \mathrm{~S}_{3}$ nanostructures with a sheaf-like morphology, which resemble the morphology some minerals assume in nature. We propose these structures form by the splitting crystal growth mechanism, as do their natural couterparts. By changing the synthetic parameters, we obtained various forms of splitting, analogous to minerals, in our case of $\mathrm{Bi}_{2} \mathrm{~S}_{3}$.

Our synthesis of the $\mathrm{Bi}_{2} \mathrm{~S}_{3}$ nanostructures is based on the reaction between bismuth carboxylate complexed with oleic acid and elemental sulfur in 1-octadecene. Two commercially available bismuth carboxylates, i.e., bismuth acetate and bismuth 2-ethylhexanoate were used and similar results were obtained. For the synthesis of $\mathrm{Bi}_{2} \mathrm{~S}_{3}$ sheaf nanostructures, $0.19 \mathrm{~g}$ bismuth acetate $(5 \mathrm{mmol})$ was added to $1 \mathrm{~g}$ oleic acid $(\sim 35 \mathrm{mmol})$ and heated to $100{ }^{\circ} \mathrm{C}$ for $10 \mathrm{~min}$ to form a colorless solution. $10 \mathrm{ml}$ 1-octadecene was then added to the bismuth-oleic acid solution, degassed, and heated to $180{ }^{\circ} \mathrm{C}$. The sulfur precursor solution was made by heating $32 \mathrm{mg}$ elemental sulfur in $5 \mathrm{ml}$ 1-octadecene at $150{ }^{\circ} \mathrm{C}$ under argon and resulted in a clear yellow solution. The sulfur solution was injected to the bismuth-oleic acid solution, and the formation of the $\mathrm{Bi}_{2} \mathrm{~S}_{3}$ nanostructures could be followed by the color change of the mixture, from light yellow to light brown, and eventually to black. The black precipitate was isolated by centrifugation and washed several times with acetone. The precipitate can be dispersed in organic solvents such as hexane, preferably with the aid of sonification. Alternatively, we also tried injection of bismutholeic acid to sulfur solution or mixed the two precursors at room temperature and then heated to $180{ }^{\circ} \mathrm{C}$; and with the same amount of precursors and solvent as mentioned above, we obtained $\mathrm{Bi}_{2} \mathrm{~S}_{3}$ sheaf nanostructures as well. However, by varying synthetic parameters such as injection temperature, bismuth/ oleic acid ratio, bismuth/sulfur ratio, we obtained $\mathrm{Bi}_{2} \mathrm{~S}_{3}$ nanostructures with different morphologies. 
O’Brien et al recently reported using bismuth acetate with corresponding elemental chalcogen in refluxing octylamine to make bismuth chalcogenides nanostructures [12], and later on extended the method to antimony chalcogenides nanostructures [18]. Nanorods of $\mathrm{Bi}_{2} \mathrm{~S}_{3}$ and hexagonal wafers of $\mathrm{Bi}_{2} \mathrm{Se}_{3}$ and $\mathrm{Bi}_{2} \mathrm{Te}_{3}$ were obtained, indicating a correlation between the crystalline structure and resulting morphology of the nanomaterials. Fang et al [19] also reported synthesis of $\mathrm{Bi}_{2} \mathrm{Te}_{3}$ hexagonal nanoplatelets with high uniformity in size and shape by a reaction between bismuth 2-hexylhexanoate and trioctylphosphine telluride in the presence of oleic acid at $150{ }^{\circ} \mathrm{C}$.

In our synthesis, with the reaction between bismuth acetate complexed with oleic acid and elemental sulfur in a noncoordinating solvent 1-octadecence, we obtained novel $\mathrm{Bi}_{2} \mathrm{~S}_{3}$ nanostructures. Figure 1A shows a representative transmission electron microscope (TEM) image of such $\mathrm{Bi}_{2} \mathrm{~S}_{3}$ nanostructures. They look like a wheat sheaf, - as if a bundle of filamentary crystals have been bandaged in its middle with the top and bottom fanning out while the middle remaining thin — hence the name "sheaf structures". The individual nanofilaments have an average diameter of $9 \mathrm{~nm}$ and the sheaves are $~ 570 \mathrm{~nm}$ in length.

Although the double-sheaf morphology dominates in our synthesis product, complex structures made of 3-6 half-sheaves originating from the same core as well as half-sheaf structures with a V-shape were also obtained. For example, Figure 1B shows a half-sheaf structure, while Figure 1C and D shows rather symmetric structures formed by the intergrowth of two or three double-sheaves from the same core. High resolution TEM analysis on the core part of the multiple-sheaf structures may be expected to provide structural information which may help understand why such structures form, however, the thickness of the core part and the strong electron scattering from bismuth has made such measurements unattainable. Recently several research groups [20-22] reported the formation of $\mathrm{MnO}$ multipods (each pod is a nanorod terminated with diamond-like arrows). These $\mathrm{MnO}$ multipods relate to the $\mathrm{Bi}_{2} \mathrm{~S}_{3}$ structures shown 
here, in that various numbers from 2 to 6 of $\mathrm{MnO}$ pods originate from one core part to form $\mathrm{T}$ shapes, crosses and hexapods. Oriented attachment was proposed for the formation of those $\mathrm{MnO}$ multipod structures in two of these reports; however, it is difficult to distinguish between shape evolution during growth and the oriented growth mechanism, therefore more detailed studies of the structures may be needed to assign a mechanism.

Figure 2 shows the powder X-ray diffraction (XRD) pattern of the sheaf structures and all the peaks can be indexed to orthorhombic $\mathrm{Bi}_{2} \mathrm{~S}_{3}$ (JCPDS 17-0320) with no indication of impurities. Figure 3 shows a selected area electron diffraction (SAED) pattern obtained on a sheaf structure. It further confirms that the sheaf structures are pure crystalline $\mathrm{Bi}_{2} \mathrm{~S}_{3}$ phase and the textured ring pattern is consistent with a bundle of filaments partially aligned along the common axis. By calibrating the rotation between the TEM image and the diffraction pattern with $\alpha-\mathrm{MoO}_{3}$, we determine the nanofilaments, of which the sheaf structures are composed, grow along the [001] direction.

High resolution TEM analysis of these sheaf structures indicates that each nanofilament is single crystalline. Figure 4 shows a representative HRTEM image of a nanofilament with its corresponding Fourier transformation.

Very interestingly, it has already been known that some minerals tend to form this seemingly unusual sheaf structure in nature. For example, stiblite, a mineral of the zeolite group, often displays the characteristic sheaf structure, and thus obtained its other name 'desmine', which means "sheaf-like" in Greek. It is believed by mineralogists that the sheaf structures form by crystal splitting during their growth. Adapted from reference [23], Figure 5A shows drawings of successive stages of splitting during crystal growth, i.e., an individual crystal splits apart, forming a number of subindividuals (simple splitting), a sheaf-like structure, or in its final form, a spherulitic structure. Different minerals are found to have a different "splitting ability", depending on their crystal structure. For example, aragonite (the orthorhombic phase of $\mathrm{CaCO}_{3}$ ) 
has a higher splitting ability than calcite (the rhombohedral phase of $\mathrm{CaCO}_{3}$ ), and therefore tend to form split acicular crystals or frostwork-like structures.

Crystal splitting may occur due to several reasons and it has not been completely understood. However, generally speaking, splitting is associated with fast crystal growth. With other conditions being the same, crystal growth depends strongly on the solution oversaturation. Punin et al. suggested [24] that splitting is only possible if the oversaturation exceeds a certain 'critical' level, which is specific for each mineral and the given conditions. Other factors that have been found to cause crystal splitting are mechanical splitting, that is when extra molecules appear in some layers of its crystallographic network and chemical splitting, that is when certain ions (e.g., $\mathrm{Mg}^{2+}$ and $\mathrm{Ca}^{2+}$ ) are present in the parent solution [23]. Depending on the level of supersaturation or impurity concentration (which can change during growth), minerals can take on different degrees of splitting, resulting in a number of subforms of split crystals. Examples of some minerals displaying different forms of splitting are shown in Figure 5B-E. Figure 5B is a picture of a quartz rod without splitting [25] while Figure $5 \mathrm{C}$ and D show the late stage of simple splitting and early stage of sheaf splitting of quartz respectively. Figure 5E shows a wavellite (an aluminum phosphate mineral) spherulite. Figure 5C-E are adapted from reference [26].

In our synthesis, we found that, by controlling synthetic parameters such as temperature, amount of oleic acid and modulation of addition of the precursors etc, various morphologies of $\mathrm{Bi}_{2} \mathrm{~S}_{3}$ can be obtained. Analogous to what have been observed in minerals, our synthesized $\mathrm{Bi}_{2} \mathrm{~S}_{3}$ can also take on different forms of splitting. In Figure 5F-I, we show the $\mathrm{Bi}_{2} \mathrm{~S}_{3}$ nanostructures in the form of individual nanorods with no splitting, of small bundles with simple splitting, of sheaf structures and finally, of spherulitic strucutures. The synthetic parameters for obtaining each structure are described in the supporting information. As already known, different minerals display different splitting ability, depending on their crystal structure. Our synthesis clearly demonstrates that $\mathrm{Bi}_{2} \mathrm{~S}_{3}$ has a strong splitting ability and this is consistent with its chain-like crystalline structure [27]. $\mathrm{Bi}_{2} \mathrm{~S}_{3}$ has an orthorhombic lattice with four molecules per unit 
cell. Each bismuth atom has three nearest-neighbor sulfur atoms at distances that are approximately the sum of the covalent radii and beyond these, there are three or four additional neighbors at distances that are not much larger. It is possible to think of this structure as made up of infinite chains of stoichiometric composition running parallel to the c-axis, and close to the [010] directions. The binding between these chains is considerably weaker than that within the chains. This suggests that cleavage may take place in the (010) planes, and preferential growth occurs in the [001] direction. Indeed, as already shown by previous literature reports, $\mathrm{Bi}_{2} \mathrm{~S}_{3}$ tends to form one-dimensional nanostructures, including nanorods, nanowires and nanoribbions [1214], or more complex structures [15-17] made up of the aforementioned basic units, including this work. Furthermore, the nanofilaments of the sheaf structures do grow along the [001] direction in our case, which provides additional support of the splitting growth mechanism of these structures.

As shown in Figure 5, the unique $\mathrm{Bi}_{2} \mathrm{~S}_{3}$ sheaf structures form only under certain conditions. The different forms of splitting shown in Figure 5F-H are varied by only changing the reaction temperature, with otherwise identical conditions (injection of sulfur to Bi-oleic acid solution). The degree of splitting decreases with increasing injection temperature, i.e., from sheaf structure at $180{ }^{\circ} \mathrm{C}$, to small bundles with simple splitting at $220^{\circ} \mathrm{C}$ and eventually to individual nanorods with no splitting at $260{ }^{\circ} \mathrm{C}$ (also resulted in a large amount of $\mathrm{Bi}$ colloidal crystals). These observations may be qualitatively explained in the context of the crystal splitting theory combined with the nucleation and growth theory of nanocrystals. In the nanocrystal synthesis, the formation of nanocrystals with non-thermodynamically equilibrium shapes is driven kinetically. According to both this and the crystal splitting theory, the formation of these sheaf structures requires fast crystal growth. From the nanocrystal synthesis point of view, the overall growth of nanocrystals is believed to occur in two steps, that is, the initial formation of nuclei just after supersaturation and subsequent growth of the nuclei. At higher temperature, more nuclei will form in a shorter time; a large number of nuclei will then grow slowly. 
The most interesting feature of crystal splitting is that new surface area is created each time the crystal splits. Thus crystal splitting is favored in a situation where the organic surfactant is a very potent surface stablizer, and where at equilibrium the balance of bulk and surface energies dictates a particular crystal size. At lower temperature, when few nuclei form, fast growth can lead to a situation of metastability, where crystals grow beyond this size. It is then thermodynamically favorable for a large crystal to split, because of the strong adhesion of the surfactant to the newly created surface.

To shed light on the formation of the splitting structures, we studied their temporal morphological evolution by taking TEM images on aliquots obtained at different time intervals from a reaction. Figure 6 show the splitting evolution of the sheaf structures. From Figure 6A and $\mathrm{B}$, we can see that splitting already occurs in the initially formed structures at very early stage. As reaction proceeds, the number of nanofilaments increases and meanwhile, the individual nanofilaments grow, but mainly in the elongated direction. Figure 7 shows the morphological evolution in the case of simple splitting. At the early stage, single units with no splitting were observed as shown in Figure 7A.

We also found that the same solution that would produce sheaf nanostructures under vigorous stirring would yield yet another striking morphology under static condition $-\mathrm{Bi}_{2} \mathrm{~S}_{3}$ nanoflowers. Figure 8 shows representative scanning electron microscopy (SEM) images of $\mathrm{Bi}_{2} \mathrm{~S}_{3}$ nanoflowers, which usually are composed of five to eight petals with all the petals originating from the same point in a highly symmetric fashion. The average length of the petals is about $900 \mathrm{~nm}$. Figure 9A shows the two-dimensional projection image of a six-petal nanoflower by TEM. Both SEM and TEM characterization reveal that each petal of a nanoflower is a bundle of nanofilaments and the tips of some individual nanfilaments can be clearly identified by TEM. High resolution TEM image, as in Figure 9B, shows that each nanofilament is single crystalline and the nanofilaments are co-aligned with the same growth direction, as evidenced by the lattice planes. The diameters of the individual nanofilaments range from about 5 to $10 \mathrm{~nm}$. 
We would like to point out that these nanoflowers show quite some analogy to the sheaf structures, and look like the three-dimensional counterparts of the sheaf structures. For example, comparing Figure 1C and 8A, we can see that the two structures show very similar motif in twodimensions. They differ, in terms of morphology, mainly in that the petals of the nanoflowers are made up of a much larger number of nanofilaments and thus form a three-dimensional cylindrical shape while the sheaf structures take on a two-dimensional, flat fan-like shape. It has been noted that numerous minerals split to form flat, fan-like sheaves instead of three-dimensional ones [26].

In the formation of the $\mathrm{Bi}_{2} \mathrm{~S}_{3}$ sheaf structures, although structures made up of two or three double-sheaves are present, the simple double-sheaf structures dominate. This trend is reversed in the formation of the $\mathrm{Bi}_{2} \mathrm{~S}_{3}$ nanoflowers, which are called so as they have at least 4 petals. Due to the same reasons mentioned earlier, we can not provide more detailed structural characterization on the core part of the nanoflowers by HRTEM, and therefore can not give further insight into why such structures form. Furthermore, without the characterization of the core part, we can not conclude if the nanoflowers are indeed the counterparts of the sheaf structures in threedimensions forming by also splitting growth, as we can not determine whether the petals are aggregates of many individual nanowires or a single crystal with nanowires splitting at its end. It should be stressed that, a split crystal is a single crystal and its subindividuals grow from a single nucleus and are not separated from each other. One speculation for the sheaves' intergrowth is twinning complexed with splitting growth. Although this is not common in minerals, it has been observed, for example, cruciform twinning complexed with splitting growth in some cases of quartz [26].

Recently, effort has been directed towards bio-inspired mineralization of various technically important inorganic crystals leading to crystals with unusual structural specialty and complexity. The $\mathrm{Bi}_{2} \mathrm{~S}_{3}$ sheaf structure reported here bear certain resemblance to some of the structures obtained in biomimetic synthesis. For example, Kniep et al [28] investigated the biomimetic growth of fluorapatite in gelatin matrices and found that starting with hexagonal prismatic seed 
crystals, dumbbell-shaped aggregates of fluorapatite grow and eventually form closed spheres. They believe the structures show close parallel to the topological branching of the macromolecular starburst dendrimers and propose the dumbbell-shaped structures formed by selfsimilarity branching. Recently, they [29-30] revisited this biomimetic mineralization process of the fluorapatite-gelatin composites and showed evidence for a direct correlation between the intrinsic electrical dipole fields and the induction of the hierarchical formation of the structures. Despite certain apparent morphology similarity between the dumbbells and our $\mathrm{Bi}_{2} \mathrm{~S}_{3}$ sheaf structures, we believe the formation mechanism differ significantly.

In summary, we report the synthesis of novel $\mathrm{Bi}_{2} \mathrm{~S}_{3}$ nanostructures with a sheaf-like morphology via a simple solution method. We propose that these structures form by the splitting crystal growth mechanism, as has been noted in some minerals in nature. By controlling the synthetic parameters, different forms of splitting are obtained in our case of $\mathrm{Bi}_{2} \mathrm{~S}_{3}$.

\section{Acknowledgements}

We would like to thank Haitao Liu, Steven Hughes and Can Erdonmez for assistance in SEM, HRTEM and Electron Diffraction respectively. This work was supported by the Director, Office of Energy Research, Office of Science, Division of Materials Sciences, of the U.S. Department of Energy under Contract No. DE-AC02-05CH00231. We also acknowledge support of the National Center for Electron Microscopy, Lawrence Berkeley Lab, which is supported by the U.S. Department of Energy under Contract No. DE-AC02-05CH11231.

\section{Supporting Information}

Materials, characterization and synthetic parameters for different of $\mathrm{Bi}_{2} \mathrm{~S}_{3}$ nanostructures. 


\section{References}

1. Y. Yin and A. P. Alivisatos. Nature 2005, 437, 664.

2. L. Manna, D. J. Milliron, A. Meisel, E. C. Scher and A. Alivisatos. Nat. Mater. 2003, 2, 382.

3. D. J. Milliron, S. M. Hughes, Y. Cui, J. B. Li, L. W. Wang and A. P. Alivisatos. Nature, 2004, 430, 190.

4. A. G. Kanaras, C. Sönnichsen, H. Liu, and A. P. Alivisatos. Nano. Lett. 2005, 5, 2164.

5. S-H. Yu and H. Cölfen. J. Mater. Chem. 2004, 14, 2124

6. L. Qi, H. Cölfen, M. Antonietti, M. Li, J. D. Hopwood, A. J. Ashley, and S. Mann. Chem. Eur. J. 2001, 7, 3526.

7. L. Qi, H. Cölfen, and M. Antonietti. Angew. Chem. Int. Ed. 2000, 39, 3604.

8. L. Qi, H. Cölfen, and M. Antonietti. Chem. Mater. 2000, 12, 2392.

9. R. Suarez, P. K. Nair, and P. V. Kamat. Langmuir 1998, 14, 3236.

10. B. Chen and C. Uher. Chem. Mater. 1997, 9, 1655.

11. Oded Rabin, J. M. Perez, J. Grimm, G. Wojtkiewicz and R. Weissleder. Nat. Mater. 2006, 5, 117.

12. P. Christian and P. O’Brien. J. Mater. Chem. 2005, 15, 3021.

13. M. B. Sigman, Jr. and B. A. Korgel. Chem. Mater. 2005, 17, 1655.

14. Z. Liu, S. Peng, Q. Xie, Z. Hu, Y. Yang, S. Zhang and Y. Qian. Adv. Mater. 2003, 15, 936.

15. C. Jiang, S-H. Yu, W-T. Yao, H. Ge, and G-Z. Zhang. Chem. Mater. 2005, 17, 6094.

16. X. Zhou, S. Chen, D. Zhang, X. Guo, W. Ding, and Y. Chen. Langmuir 2006, 22, 1383.

17. Q. Lu, F. Gao, and S. Komarneni. J. Am. Chem. Soc. 2004, 126, 54.

18. P. Christian and P. O’Brien. J. Mater. Chem. 2005, 15, 4949.

19. W. Lu, Y. Ding, Y. Chen, Z. L. Wang, and J. Fang. J. Am. Chem. Soc. 2005, 127, 10112.

20. D. Zitoun, N. Pinna, N. Frolet, and Claude Belin. J. Am. Chem. Soc. 2005, 127, 15034.

21. X. X. Zhong, R. Xie, L. Sun, I. Lieberwirth, and W. Knoll. J. Phys. Chem. 2006, 110. 2.

22. T. Ould-Ely, D. Prieto-Centurion, A. Kumar, W. Guo, W. V. Knowles, S. Asokan, M. S. Wong, I. Rusakova, A. Lüttge, and K. H. Whitmire. Chem. Mater. 2006, 18, ASAP.

23. D. P. Grigor’ev. Ontogeny of minerals. 1965, Jerusalem: Israel Program for Scientific Translations.

24. Yu. O. Punin. Crystal Splitting, Zapiski Vsesoyuznogo Mineralogicheskogo Obstctestva, part 110, no. 6, pp. 666-686 (Russian).

25. Picture taken at Simithsonian National Museum of Natural History in Washington, DC, courtesy of Rod Nave. 
26. B. Z. Kantor. 1997, Besedi o mineralakh (Discussions about minerals): Nazran, Astrel, 136 p, in Russian. Republished in English 2003, as Crystal growth and development interpreted from a mineral's present form:Mineralogical Almanac, v. 6.

27. J. Black, E.M. Conwell, L. Seigle, and C.W. Spencer. J. Phys. Chem. Solids 1957, 2, 240.

28. R. Kniep and S. Busch. Angew. Chem. 1996, 108, 2787; Angew. Chem. Int. Ed. Engl. 1996, 35, 2624.

29. H. Tlatlik, P. Simon, A. Kawska, D. Zahn, and R. Kniep. Angew. Chem. Int. Ed. 2006, 45, 1905.

30. P. Simon, D. Zahn, H. Lichte, and R. Kniep. Angew. Chem. Int. Ed. 2006, 45, 1911. 


\section{Figure Captions}

Figure1: Representative TEM images of $\mathrm{Bi}_{2} \mathrm{~S}_{3}$ sheaf nanostructures. Although the double-sheaf morphology dominates, complex structures made of 3-6 half-sheaves originating from the same core as well as half-sheaf structures with a V-shape were also obtained. The scale bars represent $100 \mathrm{~nm}$.

Figure 2: Powder XRD pattern of the sheaf nanostructures, which corresponds to the orthorhombic $\mathrm{Bi}_{2} \mathrm{~S}_{3}$ phase.

Figure 3: SAED pattern on the sheaf bundles further confirms its orthorhombic $\mathrm{Bi}_{2} \mathrm{~S}_{3}$ phase and the textured ring pattern corresponds to the orientation of the individual nanofilaments.

Figure 4: A HRTEM image of an individual nanofilament of the sheaf structures with its corresponding Fourier transformation shown in the inset.

Figure 5: Schematic illustration of crystal splitting and examples of different forms of splitting in minerals and our synthesized $\mathrm{Bi}_{2} \mathrm{~S}_{3}$ nanostructures. Figure A, adapted from reference [23], shows the successive stages of splitting during crystal growth. B-E show photographs of minerals that take on different forms of crystal splitting. Figure B shows a picture of quartz without splitting [25], while Figure C and D show the late stage of simple splitting and early stage of sheaf splitting of quartz, respectively. Figure E is a wavellite spherulite. Figure C-E are adapted from reference [26]. Figure F-I show representative TEM images of $\mathrm{Bi}_{2} \mathrm{~S}_{3}$ nanostructures with different forms of splitting, obtained by our synthesis. All the scale bars in F-I denote $100 \mathrm{~nm}$.

Figure 6: Morphological evolution in a case of $\mathrm{Bi}_{2} \mathrm{~S}_{3}$ sheaf splitting. Aliquots of solution were taken out at $27 \mathrm{sec}(\mathrm{A}), 40 \mathrm{sec}(\mathrm{B}), 80 \mathrm{sec}$ (C) and $270 \mathrm{sec}$ (D) respectively. The data show that splitting occurs at very early stage of the reaction.

Figure 7: Morphological evolution in a case of simple splitting of $\mathrm{Bi}_{2} \mathrm{~S}_{3}$. The scale bars represent $100 \mathrm{~nm}$.

Figure 8: Representative SEM images of $\mathrm{Bi}_{2} \mathrm{~S}_{3}$ nanoflowers. All the scale bars represent $500 \mathrm{~nm}$. 
Figure 9: Representive TEM images of a $\mathrm{Bi}_{2} \mathrm{~S}_{3}$ nanoflower. The high resolution image (B) shows that each petal is composed of single crystalline $\mathrm{Bi}_{2} \mathrm{~S}_{3}$ nanowires. 

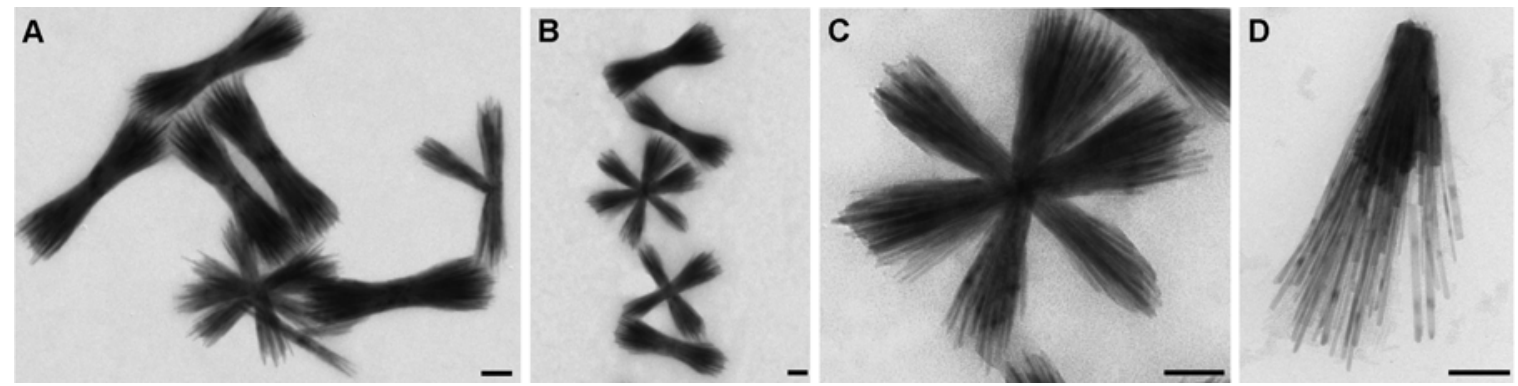

Figure1: Representative TEM images of $\mathrm{Bi}_{2} \mathrm{~S}_{3}$ sheaf nanostructures. Although the double-sheaf morphology dominates, complex structures made of 3-6 half-sheaves originating from the same core as well as half-sheaf structures with a V-shape were also obtained. The scale bars represent $100 \mathrm{~nm}$. 


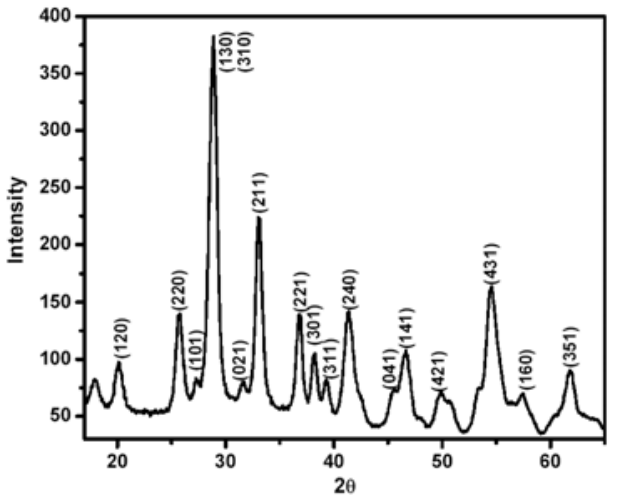

Figure 2: Powder XRD pattern of the sheaf nanostructures, which corresponds to the orthorhombic $\mathrm{Bi}_{2} \mathrm{~S}_{3}$ phase. 


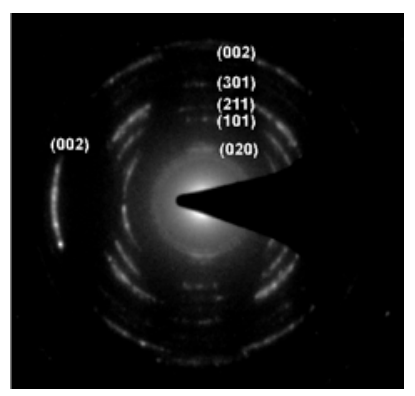

Figure 3: SAED pattern on the sheaf bundles further confirms its orthorhombic $\mathrm{Bi}_{2} \mathrm{~S}_{3}$ phase and the textured ring pattern corresponds to the orientation of the individual nanofilaments. 


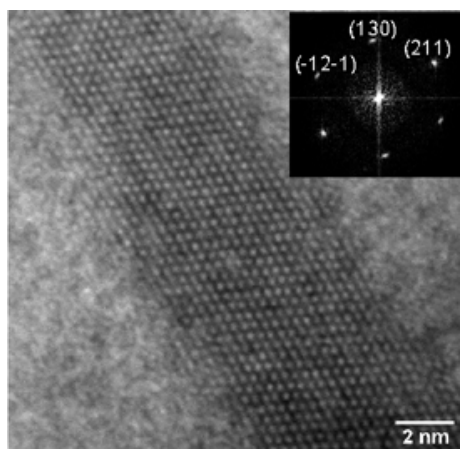

Figure 4: A HRTEM image of an individual nanofilament of the sheaf structures with its corresponding Fourier transformation shown in the inset. 
A O
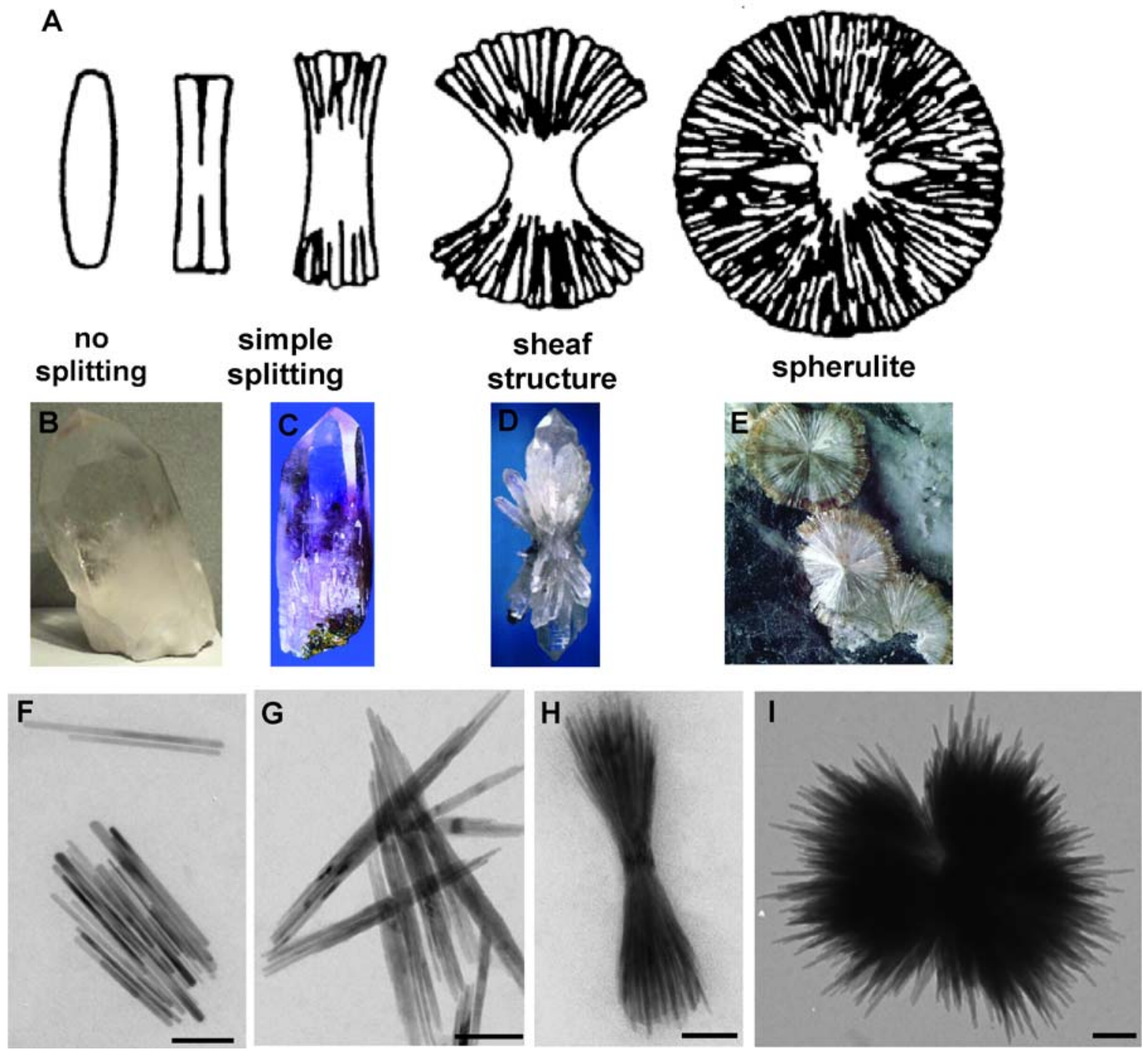

Figure 5: Schematic illustration of crystal splitting and examples of different forms of splitting in minerals and our synthesized $\mathrm{Bi}_{2} \mathrm{~S}_{3}$ nanostructures. Figure A, adapted from reference [23], shows the successive stages of splitting during crystal growth. B-E show photographs of minerals that take on different forms of crystal splitting. Figure B shows a picture of quartz without splitting [25], while Figure C and D show the late stage of simple splitting and early stage of sheaf splitting of quartz, respectively. Figure $\mathrm{E}$ is a wavellite spherulite. Figure C-E are adapted from reference [26]. Figure F-I show representative TEM images of $\mathrm{Bi}_{2} \mathrm{~S}_{3}$ nanostructures with different forms of splitting, obtained by our synthesis. All the scale bars in F-I denote $100 \mathrm{~nm}$. 

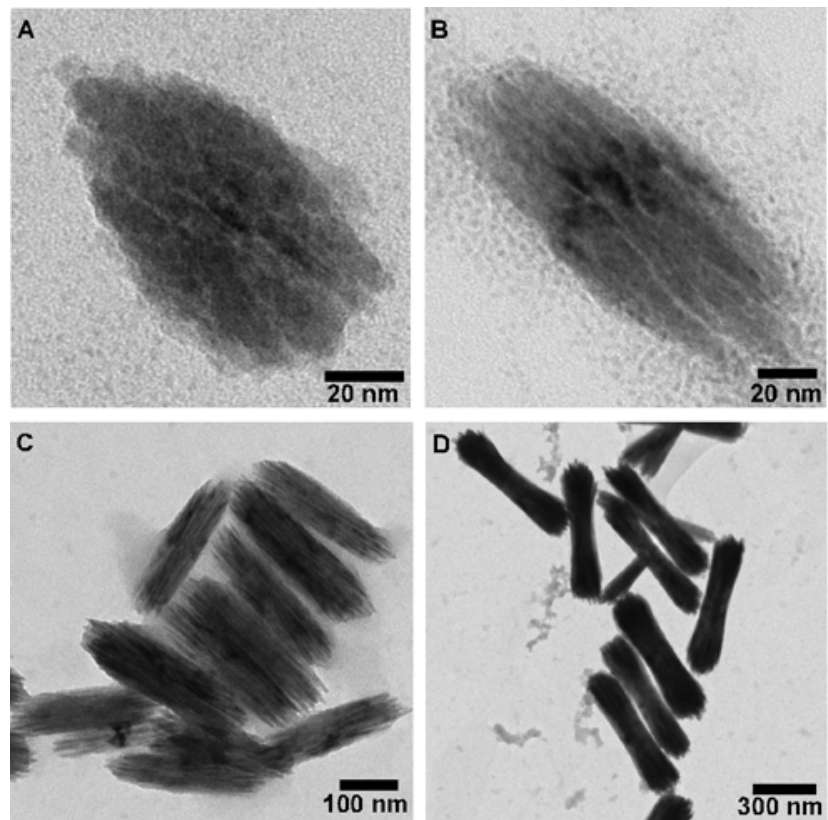

Figure 6: Morphological evolution in a case of $\mathrm{Bi}_{2} \mathrm{~S}_{3}$ sheaf splitting. Aliquots of solution were taken out at $27 \mathrm{sec}(\mathrm{A}), 40 \mathrm{sec}(\mathrm{B}), 80 \mathrm{sec}(\mathrm{C})$ and $270 \mathrm{sec}$ (D) respectively. The data show that splitting occurs at very early stage of the reaction. 

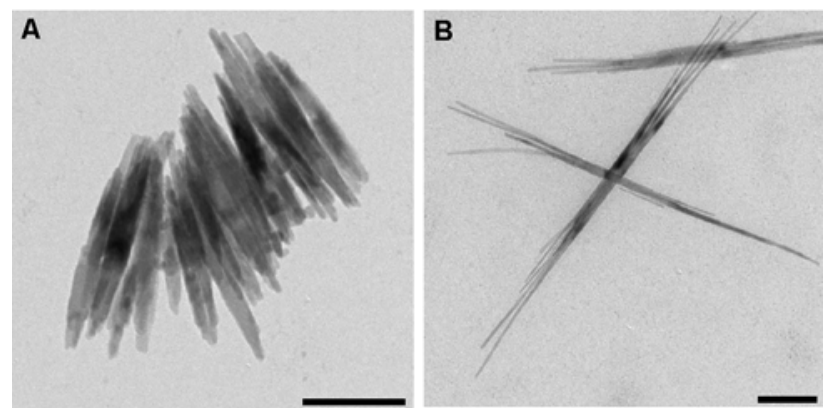

Figure 7: Morphological evolution in a case of simple splitting of $\mathrm{Bi}_{2} \mathrm{~S}_{3}$. The scale bars represent $100 \mathrm{~nm}$. 

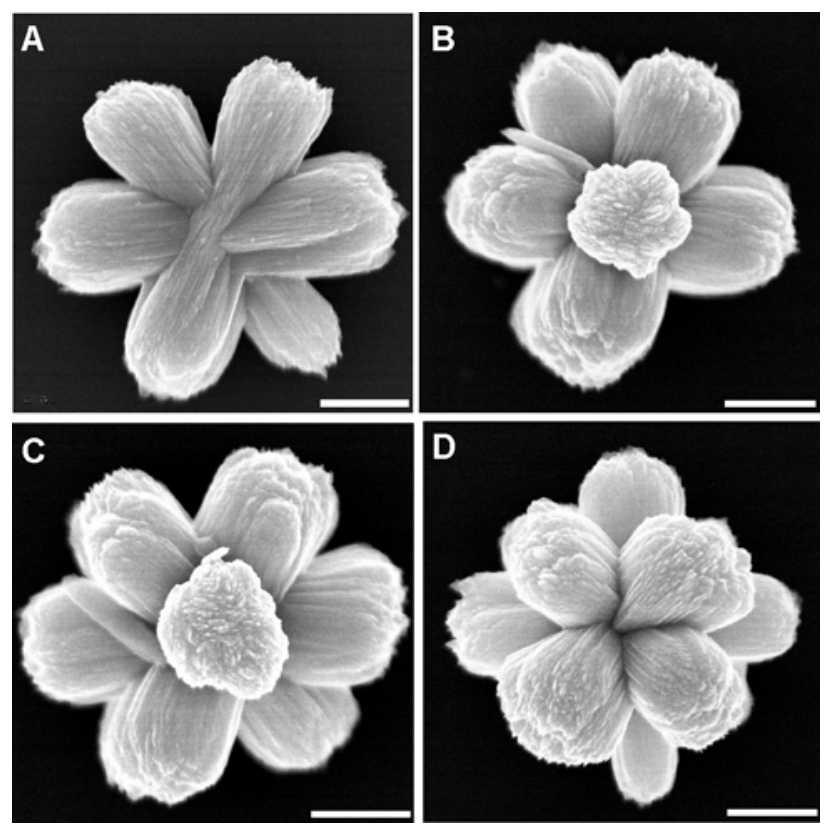

Figure 8: Representative SEM images of $\mathrm{Bi}_{2} \mathrm{~S}_{3}$ nanoflowers. All the scale bars represent $500 \mathrm{~nm}$. 

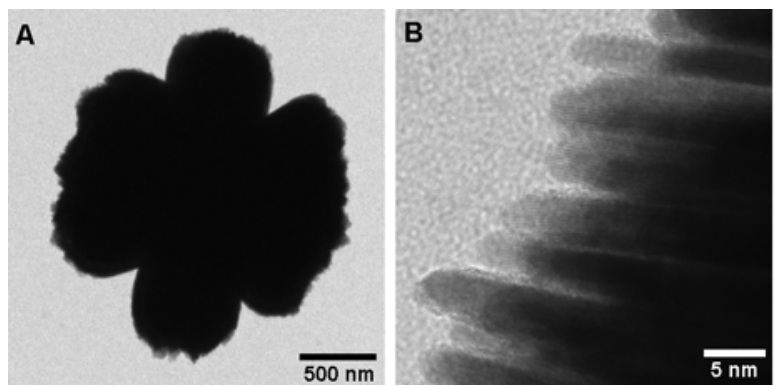

Figure 9: Representive TEM images of a $\mathrm{Bi}_{2} \mathrm{~S}_{3}$ nanoflower. The high resolution image (B) shows that each petal is composed of single crystalline $\mathrm{Bi}_{2} \mathrm{~S}_{3}$ nanowires. 